\title{
Determination of Optical Properties of Thin Films from Ketteler-Helmholtz Dispersion Relations: Application to the Case of Ultraviolet Irradiated Zirconium Oxide
}

\author{
Jean Desforges, Luc Robichaud, and Serge Gauvin \\ Groupe de Recherche sur les Couches Minces et la Photonique, Département de Physique et d'Astronomie, Université de Moncton, \\ 18 avenue Antonine-Maillet, Moncton, NB, Canada E1A 3E9 \\ Correspondence should be addressed to Serge Gauvin; serge.gauvin@umoncton.ca
}

Received 30 March 2017; Revised 5 June 2017; Accepted 19 June 2017; Published 13 August 2017

Academic Editor: Angela De Bonis

Copyright (C) 2017 Jean Desforges et al. This is an open access article distributed under the Creative Commons Attribution License, which permits unrestricted use, distribution, and reproduction in any medium, provided the original work is properly cited.

\begin{abstract}
A new method based on the Ketteler-Helmholtz dispersion relations is described. This method allows accurately determining the optical properties of thin films from a single transmittance curve. The case of zirconium oxide thin film postdeposition irradiated with ultraviolet light is analysed. The effect of ultraviolet irradiation is compared with low temperature postdeposition heating. It is shown that both processes have a comparable effect on the optical properties of the films. However, as our analysis leads to inferring that ultraviolet irradiation produces smoother film surfaces, the use of ultraviolet irradiation is an interesting alternative to the heating process. Accordingly, the reduction of light scattering from the interface with air provides films of better optical quality, which is especially valuable in case of multilayer systems.
\end{abstract}

\section{Introduction}

Novel optical materials are continuously created and the interest in regard to their optical properties is mandatory. The context of thin films is especially important [1-3]. Ideally, the theoretical formalisms regarding the determination of the complex refractive index, $\eta=n-i \kappa(\kappa>0$ for absorbing materials), should be developed with a minimal number of approximations. It is then required to compute the intensity of the reflected and/or the transmitted light out of the sample as exactly as possible. Accordingly and in order to get accurate empirical knowledge about $\eta(\lambda)$, numerous spectrophotometric methods have been developed [4]. Here, we restrict our formalism to the case of a thin film of arbitrary absorptance, deposited on a thick absorbing substrate, which is however sufficiently transparent to allow the intensity of the transmitted light to be measured with sufficient accuracy. In the present study, we apply this new approach to the case of ultraviolet irradiated zirconium oxide $\left(\mathrm{ZrO}_{2}\right)$ thin films.

Furthermore, zirconium oxide thin films have been given considerable attention in the past decades due to a wide range of possible applications, which include laser mirrors, broadband interference filters, and ionic conductors. $\mathrm{ZrO}_{2}$ is an important material, which possesses interesting properties such as large resistance against oxidation, high melting point $\left(2,715^{\circ} \mathrm{C}\right)$, excellent thermal stability, and good ionic conductivity in the Y-stabilized cubic phase. For example, $\mathrm{ZrO} 2$ thin film is a promising candidate to replace silicon dioxide as the gate dielectric in complementary metal-oxidesemiconductor technology [5-15]. It is an electrical insulating material with a band gap of $5 \mathrm{eV}$ and has potential use as an alternative material for storage capacitors in dynamic random access memories. In addition to its interesting electrical properties, $\mathrm{ZrO}_{2}$ thin film also has highly attractive optical properties such as low absorption of light, high index of refraction, high transparency over a wide spectral range, and high field damage threshold and is therefore very attractive for laser optic applications [16-23]. The properties of the film, however, highly depend on the deposition method chosen as well as the deposition conditions (substrate nature and temperature, partial gas pressure, and deposition rate). For this reason, the link between optical properties and microstructure has been widely investigated [24-29]. 
Among the various fabrication techniques available to produce thin films, vacuum depositions techniques are the most often used. The main way to produce good samples of oxide films involves a reactive process, where the metal is evaporated or sputtered in a residual atmosphere containing oxygen. In our case, $\mathrm{ZrO}_{2}$ thin films were obtained by DC reactive magnetron sputtering where argon was used as the sputtering gas in the presence of oxygen as the reactant. Sputtering deposition is a widely used technique which offers several advantages over other methods: high deposition rates; dense defect-free oxide films; not requiring high temperature; the fact that films have a composition close to that of the source material; the fact that high melting point materials are easily sputtered; sputtering usually leading to less contamination than evaporation methods; the fact that sputtered films have a good adhesion on the substrate; the fact that it can also be performed top-down. Due to its ability to reduce arcing during the reactive process, pulsed direct current magnetron sputtering is a valuable version of the sputtering technique [30-44].

Postdeposition annealing of the sample is often necessary to releases voids incorporated during the deposition process which in turn favours the formation of more closely packed thin films leading to an increase in refractive index. Postdeposition annealing is also used to complete the oxidation of the films. However, because subsidiary effects such as surface roughness modifications might come out in the process, it is important to take into account the surface morphology of the films, especially in the case of multilayer systems. The amount of energy brought into the material in such process is a key factor that leads to significant morphological differences. Indeed, the actual heating process and its temporal evolution are significant. Other postdeposition processes that can be used to complete the oxidation of the films are worth being considered and could give very different outcomes. Photonassisted oxidation has been reported to improve the quality of ultrathin (few tens of nanometres) oxide films [45-47]. These studies indicate that the rate of oxygen incorporation is enhanced by the action of ultraviolet (UV) photons by reducing the chemical activation barriers. Atomic-scale interfacial phenomena induced by ultraviolet irradiation have also been studied.

In this work, we investigate the effect of postdeposition ultraviolet irradiation on the oxidation of zirconium oxide thin films deposited by direct current reactive magnetron sputtering. We compare results obtained from postdeposition heating at relatively low temperature and ultraviolet irradiation. Both processes favour a better oxidation but our aim is to investigate how differently these processes can affect the optical and surface properties of the films.

\section{Theory}

In order to get physical understanding of the resulting properties of thin films, we use one-band Ketteler-Helmholtz dispersion relations [48] to fit theoretical transmittance spectra to the experimental ones. These dispersion relations are based on a harmonic oscillator atom (Lorentz) model and remain exact at any wavelength, even in the absorption band region and whatsoever the oscillator strength of this band. Both the real, $n$, and imaginary, $\kappa$, parts of the complex refractive index, $\eta$, can be fully determined at any wavelength, $\lambda$, through only four parameters that are physically meaningful. These parameters are $A$ : the relative permittivity (dielectric constant) on the short wavelength side of the absorption band and $B$ : the oscillator contribution to the relative permittivity (a measure of the oscillator strength) such that $A+B$ is the relative permittivity on the long wavelength side of the absorption band, the spectral location of the absorption band, $\lambda_{o}$, and finally the (approximate) relative width of this band, $\Gamma$. Formally, $\Gamma$ is a measure of the bandwidth of the absorption band. In the case of a narrow band, $\Gamma$ is the ratio of full width at half maximum of the imaginary part of the relative permittivity, $\varepsilon_{\mathrm{im}}(\lambda)$, which is equal to the product $2 n(\lambda) \kappa(\lambda)$, over $\lambda_{o}$ (the Maxwell relation, $\varepsilon=\varepsilon_{\mathrm{re}}+i \varepsilon_{\mathrm{im}}=$ $\eta^{2}$, leads to $\left.\varepsilon_{\mathrm{im}}(\lambda)=2 n(\lambda) \kappa(\lambda)\right)$. Finally, it is noteworthy that these relations satisfy the Kramers-Krönig relations [49]. Naturally, here these relations are preferred to Sellmeier's and Cauchy's dispersion relations. The one-band KettelerHelmholtz dispersion relations are

$$
\begin{aligned}
& n(\lambda)=\sqrt{\frac{1}{2}\left[\left(A+\Sigma_{A}\right)+\sqrt{\left(A+\Sigma_{A}\right)^{2}+\Sigma_{B}^{2}}\right]}, \\
& \kappa(\lambda)=\sqrt{\frac{1}{2}\left[-\left(A+\Sigma_{A}\right)+\sqrt{\left(A+\Sigma_{A}\right)^{2}+\Sigma_{B}^{2}}\right]},
\end{aligned}
$$

where

$$
\begin{aligned}
& \Sigma_{A}=B \frac{\lambda^{2}\left(\lambda^{2}-\lambda_{o}^{2}\right)}{\left(\lambda^{2}-\lambda_{o}^{2}\right)^{2}+\left(\Gamma \lambda \lambda_{o}\right)^{2}}, \\
& \Sigma_{B}=B \Gamma \frac{\lambda^{3} \lambda_{o}}{\left(\lambda^{2}-\lambda_{o}^{2}\right)^{2}+\left(\Gamma \lambda \lambda_{o}\right)^{2}} .
\end{aligned}
$$

In addition to these relations, we make use of a formalism [50] that allows computing the exact calculation of the transmittance of an arbitrary absorptance thin film, on an arbitrary absorptance substrate. The optical system of interest consists of a thin film of complex refractive index $\eta$ and of thickness $s$, deposited on a thick substrate of thickness $s_{2}$ and refractive index $\eta_{2}$. Both the incidence (labelled by the subscript 0 ) and transmission (labelled by the subscript 3 ) medium are assumed identical and transparent $\left(\eta_{3}=\eta_{0}=\right.$ $\left.n_{0}\right)$. It is possible to prevent the complication due to the internal reflections inside the substrate with the help of a wedged substrate. For a typical $1 \mathrm{~mm}$ thick substrate, a wedge angle at the interface "transmission medium|substrate," $\theta_{w}$, of about $1^{\circ}$ is sufficient. In practice, it turns out that neglecting internal reflection inside the substrate leads to very small errors. However, in the following formalism, the Fresnel coefficients include the effect of $\theta_{w}$. The incident light is assumed to be a narrow beam (ray), at normal incidence. The system is then a Fabry-Pérot resonator having a weak pseudofinesse, $F_{20}$ (we use the designation "pseudofinesse," $F_{20}$, since, formally, the usual "finesse" is a specific case of the more general concept of "reduced finesse," $\mathrm{H}_{20}$, which is 
defined by $H_{20} \equiv F_{20} / G_{20}$ (see (5)); in absence of optical losses $(\kappa=0 \Rightarrow \alpha=0)$ the reduced finesse becomes the usual finesse). Without any further significant approximation, it can be shown that the transmittance of this layered system, $t_{30}$, is given by (the rightmost index corresponds to the incidence medium)

$$
t_{30}=\gamma\left[\frac{1}{G_{20}^{2}+\left(4 / \pi^{2}\right) F_{20}^{2} \sin ^{2}(2 \pi(n s / \lambda)-\delta)}\right],
$$

with

$$
\gamma=\left\{\begin{array}{lc}
\gamma_{s}=4 \frac{n_{0} \cos \left(\theta_{3}\right)}{n_{2} \cos \left(\theta_{w}\right)}\left|\frac{\left(n_{2}-i \kappa_{2}\right) \cos \left(\theta_{w}\right)}{n_{0} \cos \left(\theta_{3}\right)+\left(n_{2}-i \kappa_{2}\right) \cos \left(\theta_{w}\right)}\right|^{2} e^{-\alpha_{2} s_{2}} & \text { for " } s \text { " type polarization } \\
\gamma_{p}=4 \frac{n_{0} \cos \left(\theta_{3}\right)}{n_{2} \cos \left(\theta_{w}\right)}\left|\frac{\left(n_{2}-i \kappa_{2}\right) \cos \left(\theta_{w}\right)}{n_{0} \cos \left(\theta_{w}\right)+\left(n_{2}-i \kappa_{2}\right) \cos \left(\theta_{3}\right)}\right|^{2} e^{-\alpha_{2} s_{2}} & \text { for " } p \text { " type polarization } \\
\text { at interface " } 3 \mid 2 ",
\end{array}\right.
$$

where the absorption coefficient, $\alpha$, is linked to the BeerLambert-Bouguer law, $I=I_{o} \exp (-\alpha z)$, and the extinction coefficient, $\kappa$, through $\alpha=4 \pi \kappa / \lambda$. The parameter $G_{20}$ describes the degradation of the finesse, due to the absorption in the thin film and the asymmetry of the interfaces " $3 / 2$ " and "1/0" [51]. Accordingly, $G_{20}$ is designated as the "reduction." The parameter $\delta$ is the mean phase shift upon internal reflections: $\delta=\left(\delta_{21}+\delta_{01}\right) / 2$. Finally, the propagation angle in the transmission medium, $\theta_{3}$, is given by $\theta_{3}=$ $\arcsin \left[n_{2} \sin \left(\theta_{w}\right) / n_{0}\right]$. The parameters $F_{20}$ and $G_{20}$ are given by $\left(r_{i j}\right.$ is the reflectance at interface " $i \mid j$ ")

$$
\begin{aligned}
F_{20} & \equiv \pi \frac{\sqrt[4]{r_{12} r_{10}}}{\sqrt{\left(1-r_{12}\right)\left(1-r_{10}\right)}}, \\
G_{20} & \equiv \frac{\exp ((1 / 2) \alpha s)-\sqrt{r_{12} r_{10}} \exp (-(1 / 2) \alpha s)}{\sqrt{\left(1-r_{12}\right)\left(1-r_{10}\right)}},
\end{aligned}
$$

with

$$
\begin{aligned}
& r_{10}=\left|\frac{\eta-n_{0}}{\eta+n_{0}}\right|^{2}, \\
& r_{12}=\left|\frac{\eta-\eta_{2}}{\eta+\eta_{2}}\right|^{2}, \\
& \delta_{10}=\arctan \left[-2 \frac{n_{0} \kappa}{\left(n^{2}-n_{0}^{2}\right)+\kappa^{2}}\right], \\
& \delta_{12}=\arctan \left[2 \frac{n \kappa_{2}-n_{2} \kappa}{\left(n^{2}-n_{2}^{2}\right)+\left(\kappa^{2}-\kappa_{2}^{2}\right)}\right] .
\end{aligned}
$$

Since the parameters $s_{2}, \eta_{2}, \theta_{w}$, and $s$ are assumed known, $t_{30}$ is determined from (3) to (6) without any further approximation and according to the actual value of $n(\lambda)$ and $\kappa(\lambda)$, which here are given by the one-band KettelerHelmholtz dispersion relations, (1).

\section{Experimental}

$\mathrm{ZrO}_{2}$ thin films (typically, $100 \mathrm{~nm}$ thick) were deposited at room temperature on a standard $1 \mathrm{~mm}$ thick soda lime glass substrate (Corning 2947 Micro Slides, $T_{g} \approx 565^{\circ} \mathrm{C}$ ) from DC reactive magnetron sputtering. The glass substrates were thoroughly cleaned using methanol and dried with nitrogen gas flow. We have used the CMS-18 deposition system from Kurt J. Lesker Co. The targets were made of zirconium, grade 702 purity, 3.0 inches in diameter, 0.25 inches in thickness. The sputtering was performed from ionization of an argon gas in the presence of oxygen (flow rate kept constant at $10 \%$ of that of argon). The total pressure of the gas mixture was also kept constant at $0.53 \mathrm{~Pa}$ ( 4 mTorr). The plasma operating power was set at $360 \mathrm{~W}$. Target to substrate distance is approximately $10 \mathrm{~cm}$, a distance roughly corresponding to the mean free path of the sputtered molecules which is an important parameter for the resulting properties of the films. To obtain uniform layer deposition, the substrate rotates with a speed of $5 \mathrm{rpm}$. The thickness of each layer was selected by controlling the deposition time.

To induce a modification of the optical properties by postdeposition heating of the films, samples were put in a standard laboratory oven (KILN MFG Inc., Colorado $\mathrm{G}$ series) running in ambient air at atmospheric pressure. During heating, the sample is sited flat on a thick glass plate in the oven. The samples were exposed for one hour to a temperature of $100^{\circ} \mathrm{C}$. Incidentally, previous studies [7, $19,24,37,39$ ] have shown that the phase morphology of the as-deposited zirconium oxide films is monoclinic when deposited by magnetron sputtering at room temperature. The monoclinic phase is known to be stable up to $1,170^{\circ} \mathrm{C}$. Then, the present low annealing temperature is well below the temperature needed to obtain the first phase transition from monoclinic to tetragonal phase. To induce a similar effect on the optical properties of the films by postdeposition ultraviolet irradiation of the films, samples were irradiated for one hour from a 300 watts' Xenon, ozone-free arc lamp (Newport 6258). The lamp specifications state that the irradiance at $0.5 \mathrm{~m}$ is $50 \mathrm{~mW}$ per $\mathrm{m}^{2}$ per $\mathrm{nm}$. Lamp to sample distance was approximately $15 \mathrm{~cm}$. We then compare the optical and morphological properties of the films under these processes. The mechanism of photon-assisted oxidation is the main hypothesis behind this part of the study. Indeed, we have chosen the wavelengths specifically in regard of their activation ability [45-47]. The fact that we observe the 


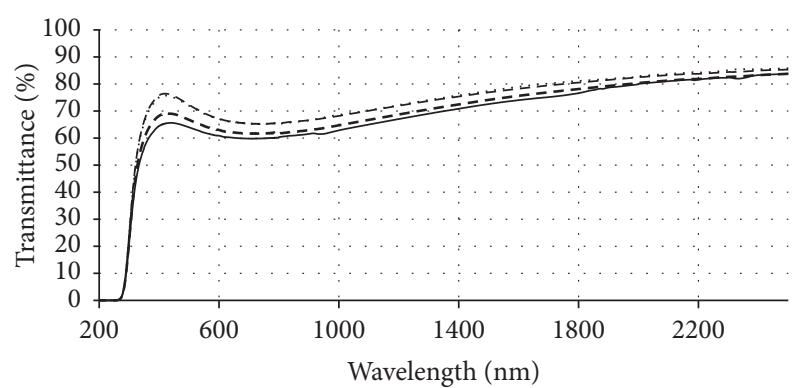

FIGURE 1: Effect of long-term UV exposure. The solid, dashed, and dotted lines correspond to as-deposited, one-hour, two-hour, and three-hour exposures, respectively. Note that there are two dotted lines that overlap.

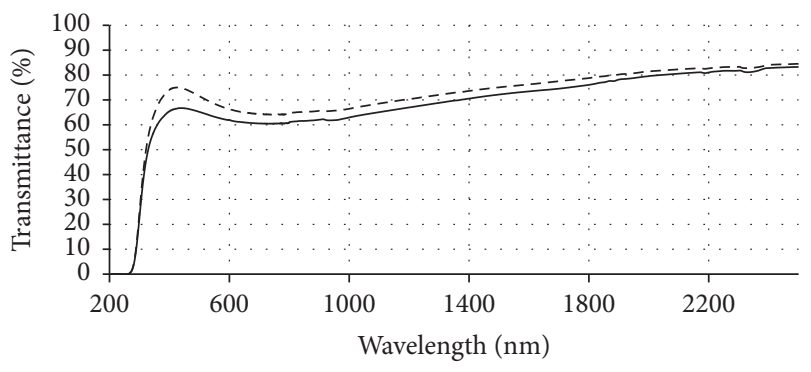

FIGURE 2: Effect of heating the sample for one hour at $100^{\circ} \mathrm{C}$.

expected increase in transmittance is a strong indication that the current interpretation is truthful. This is also supported by the fact that offset wavelength values have also been tried and result in no significant increase in transmittance.

The transmittance spectrum of the films was recorded using a spectrophotometer (CARY 5000 controlled by the Cary WinUV software) operating in the wavelength range $200-2,500 \mathrm{~nm}$. The morphological properties of the films were analysed using a Dimension 3100 Digital Instruments atomic force microscope (AFM). It operates using silicon tips with integrated cantilevers resonating at $270-340 \mathrm{kHz}$.

\section{Results}

4.1. Description. We first show how the film reacts to longterm ultraviolet exposure. In our experiments, we have set the lamp emissions at $312 \mathrm{~nm}$ and $254 \mathrm{~nm}$ because these wavelengths have a direct effect on the chemical activity of oxygen [47]. Figure 1 shows the result of three consecutive one-hour exposures with the lamp emitting at $312 \mathrm{~nm}$. The increase of transmittance is an indication of the improvement of the oxidation state of the film. One can see that the benefit of the exposure increases with time and that it was optimized after the second exposure. The third one-hour exposure did not have much effect on the sample.

It is very interesting to compare the effect of ultraviolet irradiation to what can be expected from postdeposition heating of the sample. Figures 2 and 3 show such results for one-hour ultraviolet irradiation with the lamp emitting at $254 \mathrm{~nm}$ compared to heating for one hour at $100^{\circ} \mathrm{C}$.

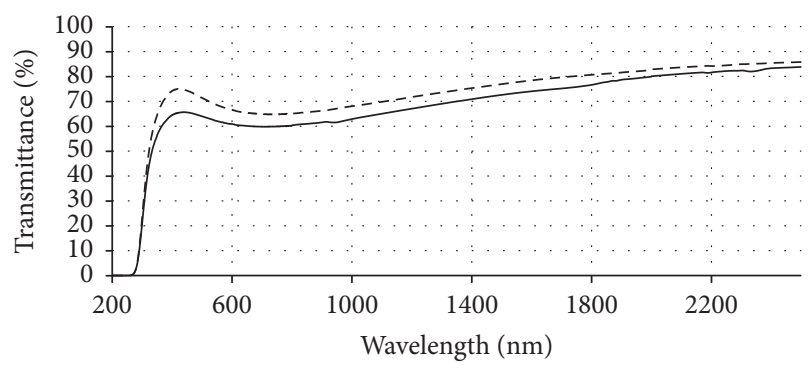

FIGURE 3: Effect of ultraviolet irradiation for one hour at $254 \mathrm{~nm}$.

One can see that the benefit of these processes in terms of improvement of the oxidation state of the films is very similar as can be seen by the increase of their respective transmittance spectrum.

In terms of surface roughness, however, the two processes could lead to very different outcomes. One of the drawbacks of postdeposition heating is that it tends to increase the surface roughness, which in turn can have a dramatic consequence on the surface diffusion and optical properties of the film. Figures 4 and 5 show the results of atomic force microscopy analysis performed on two similar samples after they have been irradiated or heated. The statistical behaviour of the surface roughness is characterized by determining the deviations of the surface profile from its mean value. The arithmetic average $R_{a}$ of the deviations, the rms value $R_{q}$, and the maximum height $R_{\max }$ are the main quantitative parameters used to characterize the surface roughness. Both Image Statistics and Box Statistics show that these parameters are indeed smaller in the case of ultraviolet irradiation. From this result, we can see that the use of ultraviolet irradiation is an interesting alternative to heating processes since it leads to less dramatic alteration of the film surface.

4.2. Spectral Analysis. Here, the parameters are $s_{2}=1 \mathrm{~mm}$, $\theta_{w}=0^{\circ}$, and $s=85 \mathrm{~nm}$ and $\eta_{2}$ is the complex refractive index of the Corning 2947 glass. The thickness of the films is determined form an a priori established calibration curve based on the deposition time. This value could appear low, but we should take into account the fact that the refractive index of all thin films is very high. In addition, the fit quality becomes unsatisfactory when $s$ deviates by more than $3 \mathrm{~nm}$.

This complex refractive index is adequately described by the following phenomenological relations (the wavelength is expressed in $\mu \mathrm{m}$ ):

$$
\begin{aligned}
& n_{2}=1.5091+0.22305 e^{-4.9882 \lambda}, \\
& \kappa_{2}=1.5 \cdot 10^{-7}+1000 e^{-75(\lambda-0.06)} .
\end{aligned}
$$

The transmittance, $t_{30}$, is determined from (3) to (6), according to the actual value of $n(\lambda)$ and $\kappa(\lambda)$, which are given by the one-band Ketteler-Helmholtz dispersion relations, (1).

Since both radiative and thermal treatments result in similar modification in transmittance spectrum and in order to characterize the largest effect obtained, our analysis is limited to the extreme cases. The first case is the lowest transmittance as-deposited thin film (see Figure 3) and the 


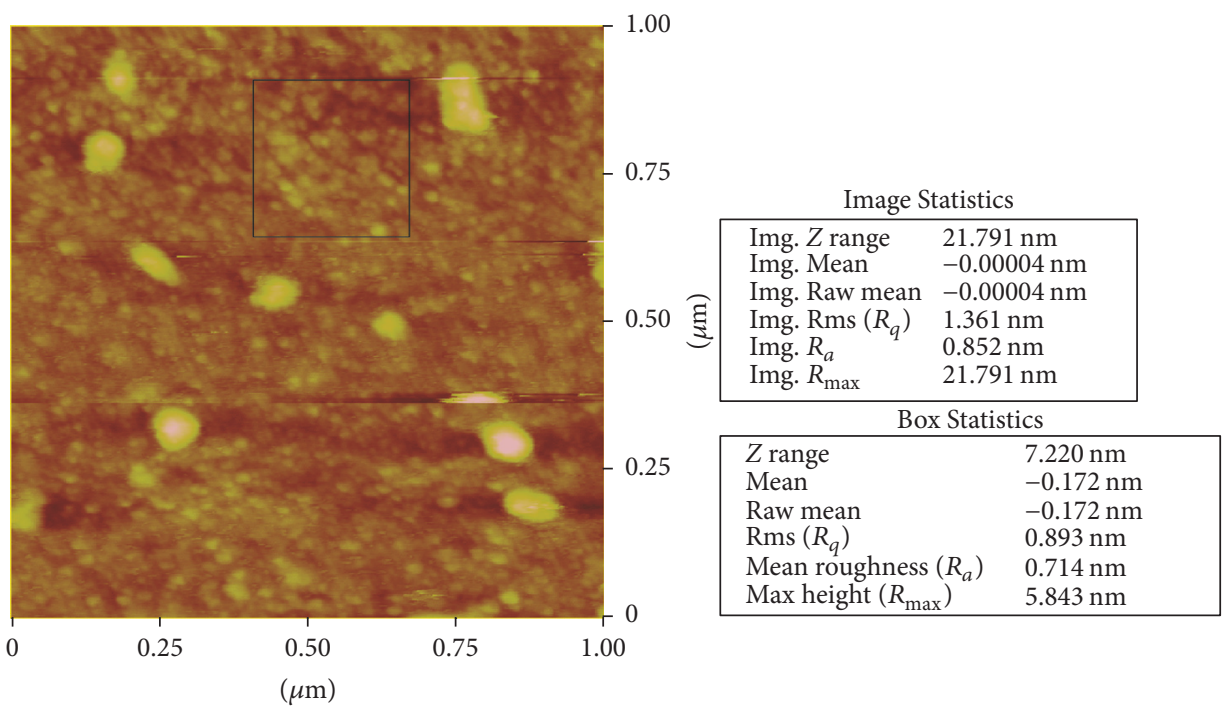

FIGURE 4: Roughness analysis of the sample heated for one hour at $100^{\circ} \mathrm{C}$.

second one is the highest transmittance UV-exposed thin film (three-hour $312 \mathrm{~nm}$ exposure; see Figure 1). Figure 6 compares the experimental spectra with the fitted spectra obtained from the above formalism.

The agreement is very good for both cases. Indeed, the curves are difficult to distinguish one from the other. From the fitted curves, we obtain the following parameters. For the as-deposited film, $A=4.6, B=0.85, \lambda_{o}=160 \mathrm{~nm}$, and $\Gamma=$ 2.5. Then, as can be seen in Figure 7 , these parameters fully determine $n(\lambda)$ and $\kappa(\lambda)$ for that film, via (1) to (2).

For the UV-exposed film, $A=4.2, B=0.9, \lambda_{o}=160 \mathrm{~nm}$, and $\Gamma=0.6$. Again and as seen in Figure 8 , these parameters fully determine $n(\lambda)$ and $\kappa(\lambda)$ for that film.

\section{Discussion}

The fact that the refractive index of the as-deposited thin film is higher than expected ( $\approx 2$ for bulk $\mathrm{ZrO}_{2}$, around $600 \mathrm{~nm}$ ) can be attributed to a partial oxidation of $\mathrm{Zr}$ in the thin film. Indeed and in accordance with the Kramers-Krönig relations, a significant metallic content increases the effective refractive index at the expense of low transmittance, as seen in all spectra presented in this paper. Eventually, this might represent a film of nonstoichiometric nature. As shown in Figure $7(\mathrm{~b})$, this assumption of a nonfully oxidized film is supported by the relatively high values for $\kappa$, which are not representative of a transparent medium. To a lesser extent and as seen in Figure 8, this lack of complete oxidation also manifests in the case of UV-exposed thin film, even though $n(\lambda)$ and $\kappa(\lambda)$ are significantly lower in the case. As an extrapolation, we could expect a similar condition for the case of heated films.

Besides, in the case of the as-deposited film, $n(\lambda)$ exhibits an unusual behaviour. Indeed, for transparent materials the refractive index usually increases with decreasing wavelength, which is the reverse situation here. However, this unusual behaviour is theoretically obtainable from the
Ketteler-Helmholtz dispersion relations when the bandwidth of the absorption band is very large. Actually, any loss mechanism can be associated with an effective "absorption" band. Accordingly, being a diffusion process that might cover a large spectral range, light scattering form the surface or the volume of thin film can be described by means of a large bandwidth absorption band, especially at short wavelengths, as it is the case here in the UV-Vis. Thus, we can expect that this is the situation for the as-deposited thin film and that UV irradiation significantly reduces light scattering. This assumption is strongly supported by the large difference in $\Gamma$ when comparing as-deposited and UV-exposed films. Since the surface roughness ("Box Statistics" in figures) is lower for irradiated film, we conjecture that it is the surface scattering that is reduced. In addition, it is worth noting that in this last case $n(\lambda)$ exhibit the usual and expected behaviour. This strongly suggests that a better oxidation of $\mathrm{Zr}$ is also achieved but apparently not completed as suggested from the higher than expected refractive index. This better oxidation could also explain the reduction of the volume scattering. Again and as an extrapolation, we could expect this to apply also in the case of heated films.

\section{Conclusions}

The introduction of a spectral analysis method based on the Ketteler-Helmholtz dispersion relations is found useful to determine the optical properties of thin films. The case of zirconium oxide appears especially interesting. Indeed, we have studied the effect of postdeposition ultraviolet irradiation on the oxidation of zirconium oxide $\left(\mathrm{ZrO}_{2}\right)$ thin films deposited by direct-pulsed current reactive magnetron sputtering and have compared this effect with low temperature postdeposition heating on samples that were not completely oxidized. We showed that the benefit of these processes in terms of improvement of the oxidation state of the films is very similar as can be seen by the increase of their 


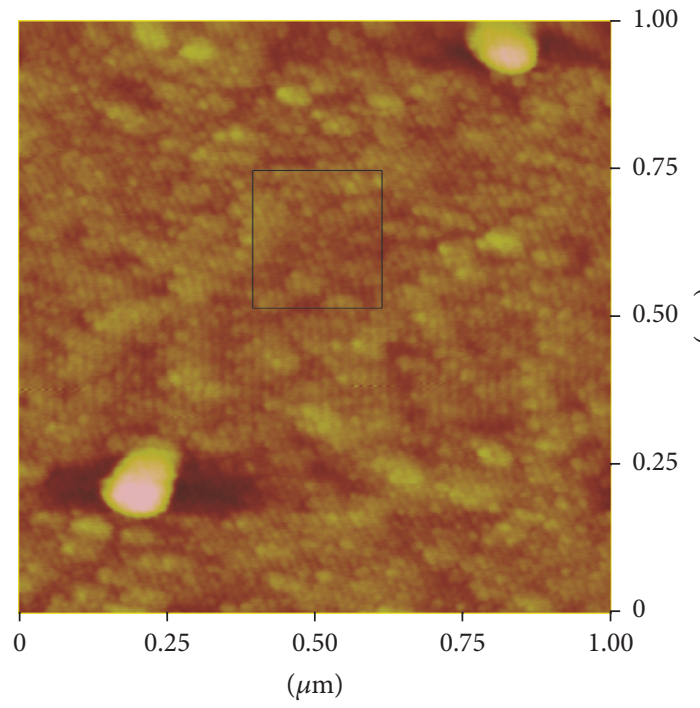

\begin{tabular}{|c|c|c|}
\hline \multicolumn{3}{|c|}{ Image Statistics } \\
\hline Img. $Z$ range & \multicolumn{2}{|c|}{$17.539 \mathrm{~nm}$} \\
\hline Img. Mean & \multicolumn{2}{|c|}{$0.000007 \mathrm{~nm}$} \\
\hline Img. Raw mean & \multicolumn{2}{|c|}{$0.000007 \mathrm{~nm}$} \\
\hline Img. Rms $\left(R_{q}\right)$ & \multicolumn{2}{|c|}{$1.336 \mathrm{~nm}$} \\
\hline Img. $R_{a}$ & \multicolumn{2}{|c|}{$0.804 \mathrm{~nm}$} \\
\hline Img. $R_{\max }$ & $17539 \mathrm{~nm}$ & $\mathrm{~nm}$ \\
\hline \multicolumn{3}{|c|}{ Box Statistics } \\
\hline$Z$ range & & $4.483 \mathrm{~nm}$ \\
\hline Mean & & $-0.148 \mathrm{~nm}$ \\
\hline Raw mean & & $-0.148 \mathrm{~nm}$ \\
\hline $\operatorname{Rms}\left(R_{q}\right)$ & & $0.614 \mathrm{~nm}$ \\
\hline Mean roughness & $\left(R_{a}\right)$ & $0.497 \mathrm{~nm}$ \\
\hline Max height $\left(R_{\mathrm{ma}}\right.$ & & $4.061 \mathrm{~nm}$ \\
\hline
\end{tabular}

FIGURE 5: Roughness analysis of the sample irradiated for one hour at $254 \mathrm{~nm}$.

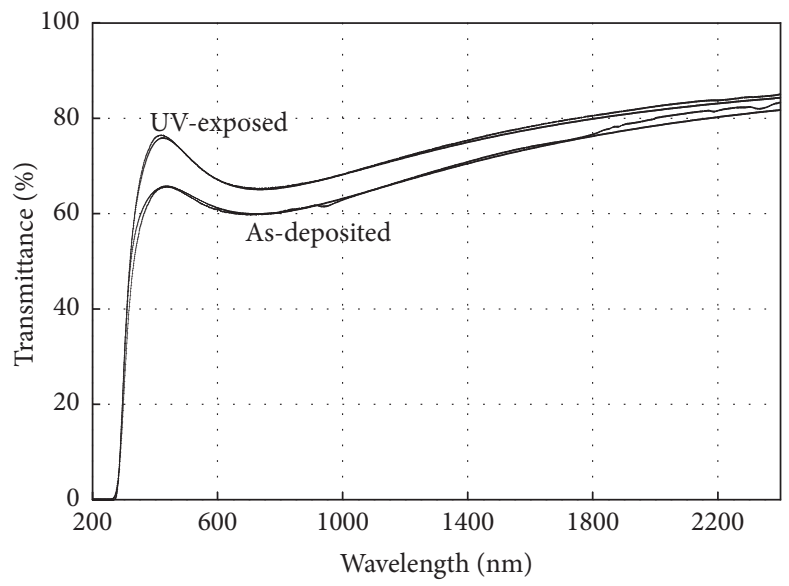

FIGURE 6: Comparison of experimental spectra with theory.

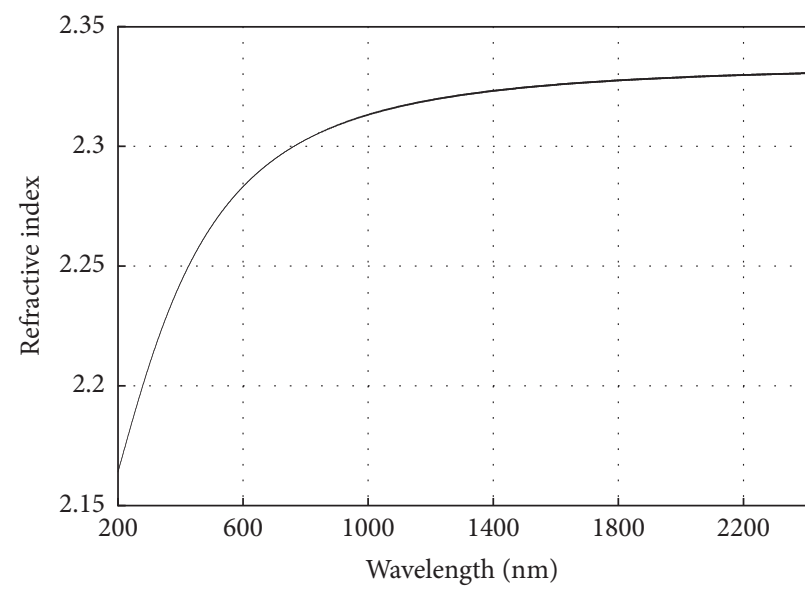

(a)

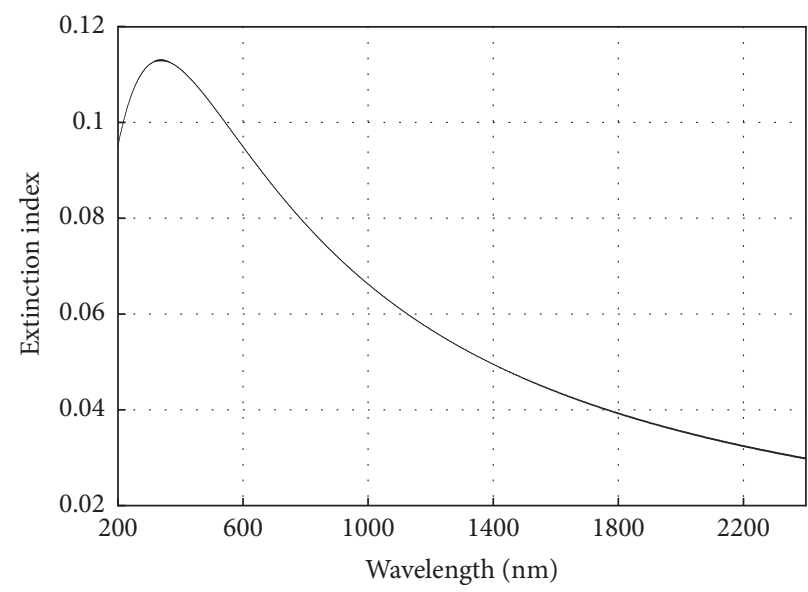

(b)

FIGURE 7: Comparison of the real (a) and imaginary (b) parts of the complex refractive index for the as-deposited thin film (see Figure 6). 


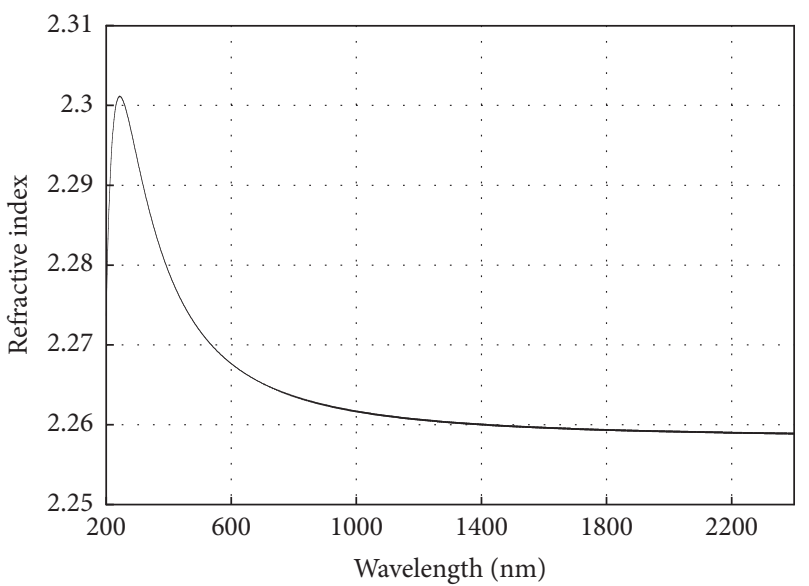

(a)

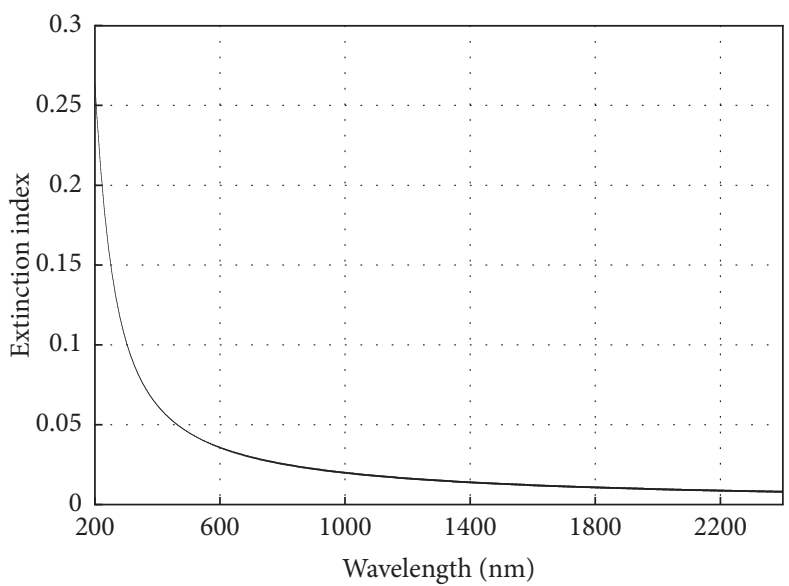

(b)

FIgURE 8: Comparison of the real (a) and imaginary (b) parts of the complex refractive index for the UV-exposed thin film (see Figure 6).

respective transmittance spectrum. In addition, the spectral analysis method described in this paper leads to identifying unusual dispersion behaviour. We infer that such behaviour can be attributed to the presence of an effective "absorption" band, which is due to a light scattering process covering a large spectral range. It seems that UV irradiation significantly reduces surface light scattering.

Besides, the evolution of the surface roughness in regard of the postdeposition processes is a key factor, depending on which process is used. Indeed, any heating process performed on thin films tends to increase their surface roughness, which in turn can have a dramatic consequence on their optical properties. We have then performed atomic force microscopy measurements on samples that have been irradiated or heated. It was shown that their respective quantitative roughness parameters are indeed smaller in the case of ultraviolet irradiation. Our results show that the use of ultraviolet irradiation is an interesting alternative to heating processes since it leads to less dramatic alteration of the film surface. This opens the way to additional treatments in order to obtain the best optical performances form thin films. Perspectives of further work include the study of the synergy between heating processes (in situ and/or ex situ) and irradiation. In addition, the relative contributions to light scattering from the surface and from the volume of thin films should also be further investigated.

\section{Conflicts of Interest}

The authors declare that there are no conflicts of interest regarding the publication of this paper.

\section{Acknowledgments}

The authors greatly thank the Fondation de l'Innovation $d u$ Nouveau-Brunswick and the Faculté des Études Supérieures et de la Recherche de l'Université de Moncton for their financial support.

\section{References}

[1] H. A. Macleod, Thin-Film Optical Filter, Taylor and Francis Group, Boca Raton, Fla, USA, 4th edition, 2010.

[2] A. Piegari and F. Flory, Optical Thin Films and Coatings: from Materials to Applications, Woodhead Publishing, Elsevier, Cambridge, UK, 2013.

[3] O. Stenzel, Optical Coatings: Materials Aspects in Theory and Practice, Springer-Verlag, Berlin, Germany, 2014.

[4] D. Poelman and P. F. Smet, "Methods for the determination of the optical constants of thin films from single transmission measurements: A critical review," Journal of Physics D: Applied Physics, vol. 36, no. 15, pp. 1850-1857, 2003.

[5] C. M. Perkins, B. B. Triplett, P. C. McIntyre, K. C. Saraswat, and E. Shero, "Thermal stability of polycrystalline silicon electrodes on $\mathrm{ZrO}_{2}$ gate dielectrics," Applied Physics Letters, vol. 81, no. 8, pp. 1417-1419, 2002.

[6] S. H. Jeong, I. S. Bae, Y. S. Shin, S.-B. Lee, H.-T. Kwak, and J.-H. Boo, "Physical and electrical properties of $\mathrm{ZrO}_{2}$ and $\mathrm{YSZ}$ highk gate dielectric thin films grown by RF magnetron sputtering," Thin Solid Films, vol. 475, no. 1-2, pp. 354-358, 2005.

[7] J. Wang, L. Zhao, N. H. Luu, D. Wang, and H. Nakashima, "Structural and electrical properties of $\mathrm{Zr}$ oxide film for high$\mathrm{k}$ gate dielectrics by using electron cyclotron resonance plasma sputtering," Applied Physics A, vol. 80, no. 8, pp. 1781-1787, 2005.

[8] M. Zhu, P. Chen, R. K. Y. Fu, W. Liu, C. Lin, and P. K. $\mathrm{Chu}$, "Microstructure and electrical properties of $\mathrm{Al}_{2} \mathrm{O}_{3}-\mathrm{ZrO}_{2}$ composite films for gate dielectric applications," Thin Solid Films, vol. 476, no. 2, pp. 312-316, 2005.

[9] M. Bizarro, J. C. Alonso, and A. Ortiz, "ZrAlO ternary oxide as a candidate for high-k dielectrics," Materials Science in Semiconductor Processing, vol. 9, no. 6, pp. 1090-1096, 2006.

[10] D. Ceresoli and D. Vanderbilt, "Structural and dielectric properties of amorphous $\mathrm{ZrO}_{2}$ and $\mathrm{HfO}_{2}$," Physical Review B Condensed Matter and Materials Physics, vol. 74, no. 12, Article ID 125108, 2006.

[11] M. Filipescu, N. Scarisoreanu, V. Craciun et al., "High-k dielectric oxides obtained by PLD as solution for gates dielectric in MOS devices," Applied Surface Science, vol. 253, no. 19, pp. 8184-8191, 2007. 
[12] F. Sacconi, J. M. Jancu, M. Povolotskyi, and A. Di Carlo, "Full-band tunneling in high- $\kappa$ dielectric MOS structures," Microelectronics Reliability, vol. 47, no. 4-5, pp. 694-696, 2007.

[13] J. Yan, Y. Kuo, and J. Lu, "Zirconium-doped hafnium oxide high-k dielectrics with subnanometer equivalent oxide thickness by reactive sputtering," Electrochemical and Solid-State Letters, vol. 10, no. 7, pp. H199-H202, 2007.

[14] S. X. Lao, R. M. Martin, and J. P. Chang, "Plasma enhanced atomic layer deposition of $\mathrm{HfO}_{2}$ and $\mathrm{ZrO}_{2}$ high-k thin films," Journal of Vacuum Science and Technology A, vol. 23, no. 3, pp. 488-496, 2005.

[15] L.-M. Chen, Y.-S. Lai, and J. S. Chen, "Influence of predeposition treatments on the interfacial and electrical characteristics of $\mathrm{ZrO}_{2}$ gate dielectrics," Thin Solid Films, vol. 515, no. 7-8, pp. 3724-3729, 2007.

[16] S. B. Qadri, C. M. Gilmore, C. Quinn, E. F. Skelton, and C. R. Gossett, "Phase stability of $\mathrm{ZrO}_{2}-\mathrm{Al}_{2} \mathrm{O}_{3}$ thin films deposited by magnetron sputtering," Physical Review B, vol. 39, no. 9, pp. 6234-6237, 1989.

[17] Q. Zhang, X. Li, J. Shen, G. Wu, J. Wang, and L. Chen, “ $\mathrm{ZrO}_{2}$ thin films and $\mathrm{ZrO}_{2} / \mathrm{SiO}_{2}$ optical reflection filters deposited by sol-gel method," Materials Letters, vol. 45, no. 6, pp. 311-314, 2000.

[18] D. Zhang, J. Shao, D. Zhang, S. Fan, T. Tan, and Z. Fan, "Employing oxygen-plasma posttreatment to improve the laserinduced damage threshold of $\mathrm{ZrO}_{2}$ films prepared by the electron-beam evaporation method," Optics Letters, vol. 29, no. 24, pp. 2870-2872, 2004.

[19] S. G. Wu, H. Y. Zhang, G. L. Tian, Z. L. Xia, J. D. Shao, and Z. $\mathrm{X}$. Fan, " $\mathrm{Y}_{2} \mathrm{O}_{3}$ stabilized $\mathrm{ZrO}_{2}$ thin films deposited by electron beam evaporation: Structural, morphological characterization and laser induced damage threshold," Applied Surface Science, vol. 253, no. 3, pp. 1561-1565, 2006.

[20] L. Liang, Y. Xu, L. Zhang, Y. Sheng, D. Wu, and Y. Sun, "Annealing effect on the optical properties and laser-induced damage resistance of solgel-derived $\mathrm{ZrO}_{2}$ films," Journal of the Optical Society of America B, vol. 24, no. 5, pp. 1066-1074, 2007.

[21] L. Liang, Y. Xu, D. Wu, and Y. Sun, "A simple sol-gel route to $\mathrm{ZrO} 2$ films with high optical performances," Materials Chemistry and Physics, vol. 114, no. 1, pp. 252-256, 2009.

[22] Y. J. Guo, X. T. Zu, B. Y. Wang, X. D. Jiang, X. D. Yuan, and H. B. Lv, "Preparation of sol-gel $\mathrm{ZrO} 2-\mathrm{SiO} 2$ highly reflective multilayer films and laser-induced damage threshold characteristic," Optik, vol. 122, no. 13, pp. 1140-1142, 2011.

[23] X. Li, X. Liu, Y. Zhao, J. Shao, and Z. Fan, "Laser-conditioning mechanism of $\mathrm{ZrO}_{2} / \mathrm{SiO}_{2}$ HR films with fitting damage probability curves of laser-induced damage," Chinese Optics Letters, vol. 8, no. 6, pp. 598-600, 2010.

[24] F. Stetter, R. Esselborn, N. Harder, M. Friz, and P. Tolles, "New materials for optical thin films," Applied Optics, vol. 15, no. 10, pp. 2315-2317, 1976.

[25] E. Ritter, "Optical film materials and their applications," Applied Optics, vol. 15, no. 10, pp. 2318-2327, 1976.

[26] D. Smith and P. Baumeister, "Refractive index of some oxide and fluoride coating materials," Applied Optics, vol. 18, no. 1, pp. 111115, 1979.

[27] J. D. T. Kruschwitz and W. T. Pawlewicz, "Optical and durability properties of infrared transmitting thin films," Applied Optics, vol. 36, no. 10, pp. 2157-2159, 1997.

[28] D. Mergel and M. Jerman, "Density and refractive index of thin evaporated films," Chinese Optics Letters, vol. 8, pp. 67-72, 2010.
[29] A. Portinha, V. Teixeira, J. Carneiro, M. F. Costa, N. P. Barradas, and A. D. Sequeira, "Stabilization of $\mathrm{ZrO}_{2}$ PVD coatings with $\mathrm{Gd}_{2} \mathrm{O}_{3}$," Surface and Coatings Technology, vol. 188-189, no. 1-3, pp. 107-115, 2004.

[30] M. H. Suhail, G. M. Rao, and S. Mohan, "Studies on the properties of zirconia films prepared by direct current reactive magnetron sputtering," Journal of Vacuum Science and Technology A, vol. 9, no. 5, pp. 2675-2677, 1991.

[31] M. S. Wong, W. J. Chia, P. Yashar, J. M. Schneider, W. D. Sproul, and S. A. Barnett, "High-rate reactive d.c. magnetron sputtering of $\mathrm{ZrO}_{x}$ coatings," Surface and Coatings Technology, vol. 86-87, no. 1, pp. 381-387, 1996.

[32] W. D. Sproul, M. E. Graham, M.-S. Wong, and P. J. Rudnik, "Reactive d.c. magnetron sputtering of the oxides of $\mathrm{Ti}, \mathrm{Zr}$, and Hf," Surface and Coatings Technology, vol. 89, no. 1-2, pp. 10-15, 1997.

[33] P. Yashar, J. Rechner, M. S. Wong, W. D. Sproul, and S. A. Barnett, "High-rate reactive sputtering of yttria-stabilized zirconia using pulsed d.c. power," Surface and Coatings Technology, vol. 94-95, pp. 333-338, 1997.

[34] K. Koski, J. Hölsä, and P. Juliet, "Properties of zirconium oxide thin films deposited by pulsed reactive magnetron sputtering," Surface and Coatings Technology, vol. 120-121, pp. 303-312, 1999.

[35] B. Hobein, F. Tietz, D. Stöver, M. Cekada, and P. Panjan, "DC sputtering of yttria-stabilised zirconia films for solid oxide fuel cell applications," Journal of the European Ceramic Society, vol. 21, no. 10-11, pp. 1843-1846, 2001.

[36] P. Gao, L. J. Meng, M. P. Dos Santos, V. Teixeira, and M. Andritschky, "Study of $\mathrm{ZrO}_{2} / \mathrm{Al}_{2} \mathrm{O}_{3}$ multilayers," Vacuum, vol. 64, no. 3-4, pp. 267-273, 2002.

[37] S. Venkataraj, O. Kappertz, H. Weis, R. Drese, R. Jayavel, and M. Wuttig, "Structural and optical properties of thin zirconium oxide films prepared by reactive direct current magnetron sputtering," Journal of Applied Physics, vol. 92, no. 7, pp. 3599_ 3607, 2002.

[38] J. M. Ngaruiya, O. Kappertz, S. H. Mohamed, and M. Wuttig, "Structure formation upon reactive direct current magnetron sputtering of transition metal oxide films," Applied Physics Letters, vol. 85, no. 5, pp. 748-750, 2004.

[39] S. Venkataraj, O. Kappertz, C. Liesch, R. Detemple, R. Jayavel, and M. Wuttig, "Thermal stability of sputtered zirconium oxide films," Vacuum, vol. 75, no. 1, pp. 7-16, 2004.

[40] D. H. Trinh, T. Kubart, T. Nyberg, M. Ottosson, L. Hultman, and H. Högberg, "Direct current magnetron sputtering deposition of nanocomposite alumina - zirconia thin films," Thin Solid Films, vol. 516, no. 23, pp. 8352-8358, 2008.

[41] D. Pamu, K. Sudheendran, M. G. Krishna, K. C. J. Raju, and A. K. Bhatnagar, "Ambient temperature stabilization of crystalline zirconia thin films deposited by direct current magnetron sputtering," Thin Solid Films, vol. 517, no. 5, pp. 1587-1591, 2009.

[42] P. J. Martin and A. Bendavid, "Properties of zirconium oxide films prepared by filtered cathodic vacuum arc deposition and pulsed DC substrate bias," Thin Solid Films, vol. 518, no. 18, pp. 5078-5082, 2010.

[43] R. Yusoh, M. Horprathum, P. Eiamchai, S. Chanyawadee, and K. Aiempanakit, "Determination of the thickness and optical constant of $\mathrm{ZrO}_{2}$ by spectroscopic ellipsometry and spectrophotometric method," Procedia Engineering, vol. 8, pp. 223-227, 2011.

[44] R. Yusoh, M. Horprathum, P. Eiamchai, P. Chindaudom, and K. Aiempanakit, "Determination of optical and physical properties 
of $\mathrm{ZrO}_{2}$ films by spectroscopic ellipsometry," Procedia Engineering, vol. 32, pp. 745-751, 2012.

[45] S. Ramanathan, G. D. Wilk, D. A. Muller, C.-M. Park, and P. C. McIntyre, "Growth and characterization of ultrathin zirconia dielectrics grown by ultraviolet ozone oxidation," Applied Physics Letters, vol. 79, no. 16, pp. 2621-2623, 2001.

[46] S. Ramanathan, P. C. McIntyre, J. Luning, P. Pianetta, and D. A. Muller, "Structural studies of ultrathin zirconia dielectrics," Philosophical Magazine Letters, vol. 82, no. 9, pp. 519-528, 2002.

[47] M. Tsuchiya and S. Ramanathan, "Effect of photon irradiation on structure of yttria-doped zirconia thin films grown on semiconductor substrates," Applied Physics Letters, vol. 91, no. 25, Article ID 253104, 2007.

[48] G. Bruhat, Optique, Section 236, Masson and Cie, 6th edition, 1965.

[49] V. Lucarini, J. J. Saarinen, K.-E. Peiponen, and E. M. Vartiainen, "Kramers-Krönig Relations in Optical Materials Research," Springer, 2005.

[50] S. Gauvin and J. Desforges, "A Self-Consistent Method for the Determination of the Complex Refractive Index of Arbitrary Absorptance Thin Films," in Proceedings of the Frontiers in Optics, p. JTh2A.107, Rochester, New York, 2016.

[51] S. Gauvin, "Accurate computation of the Briot-Sellmeier and Briot-Cauchy chromatic dispersion coefficients from the transmittance spectrum of thin films of arbitrary absorptance," Journal of the Optical Society of America A, vol. 19, no. 8, pp. 1712-1720, 2002. 

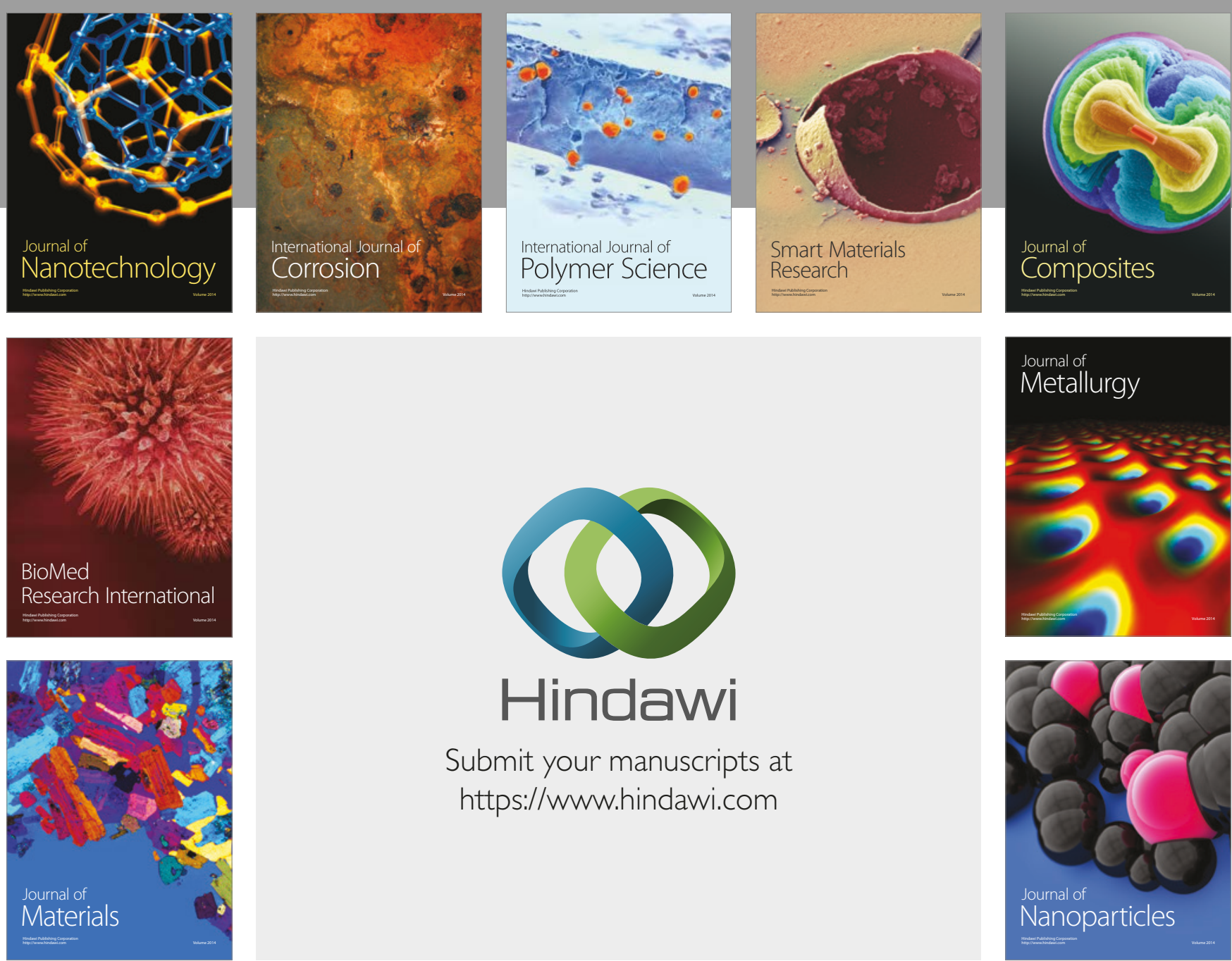

\section{Hindawi}

Submit your manuscripts at

https://www.hindawi.com
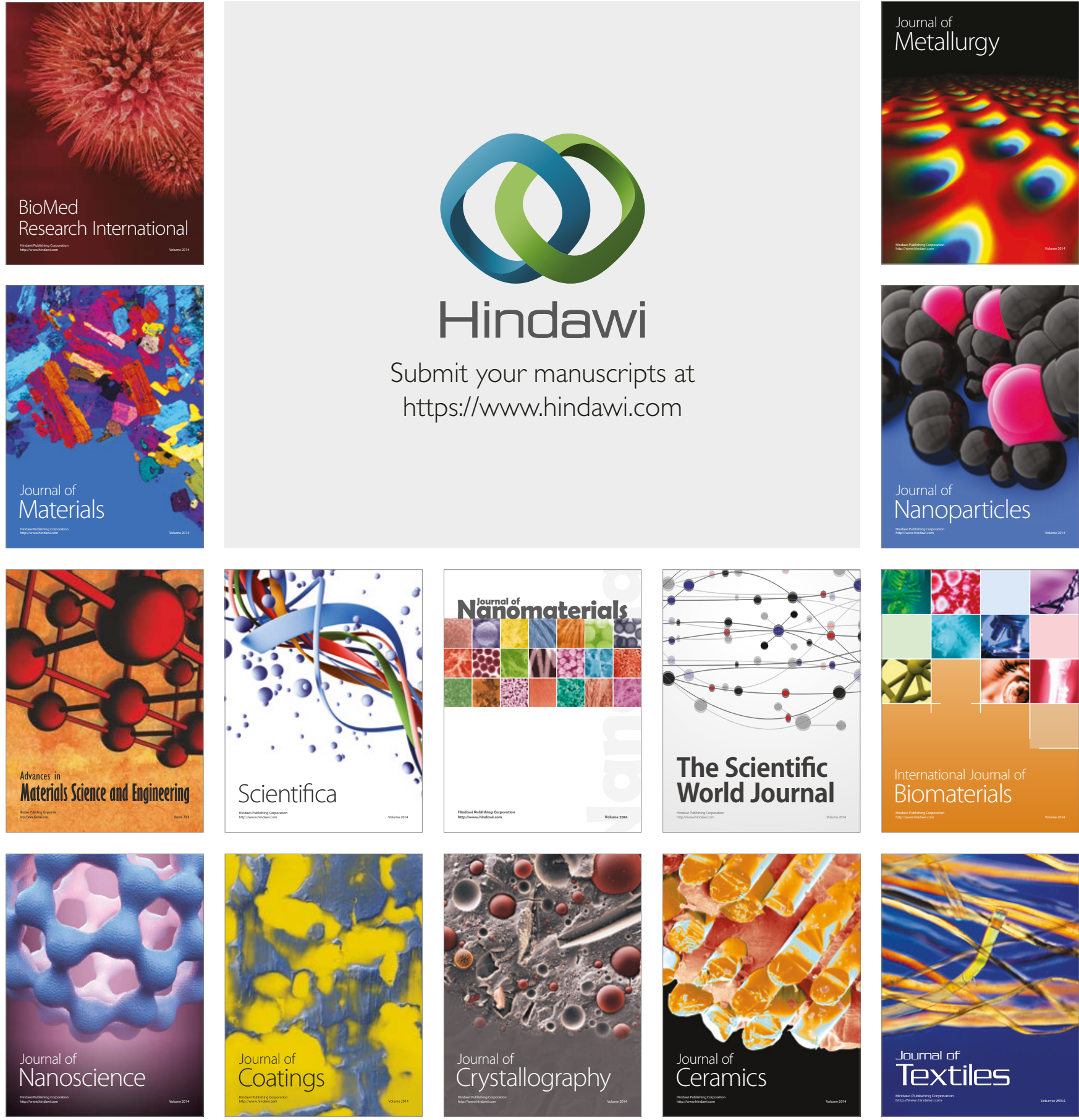

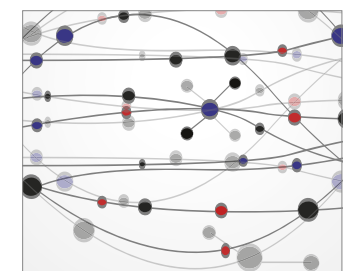

The Scientific World Journal
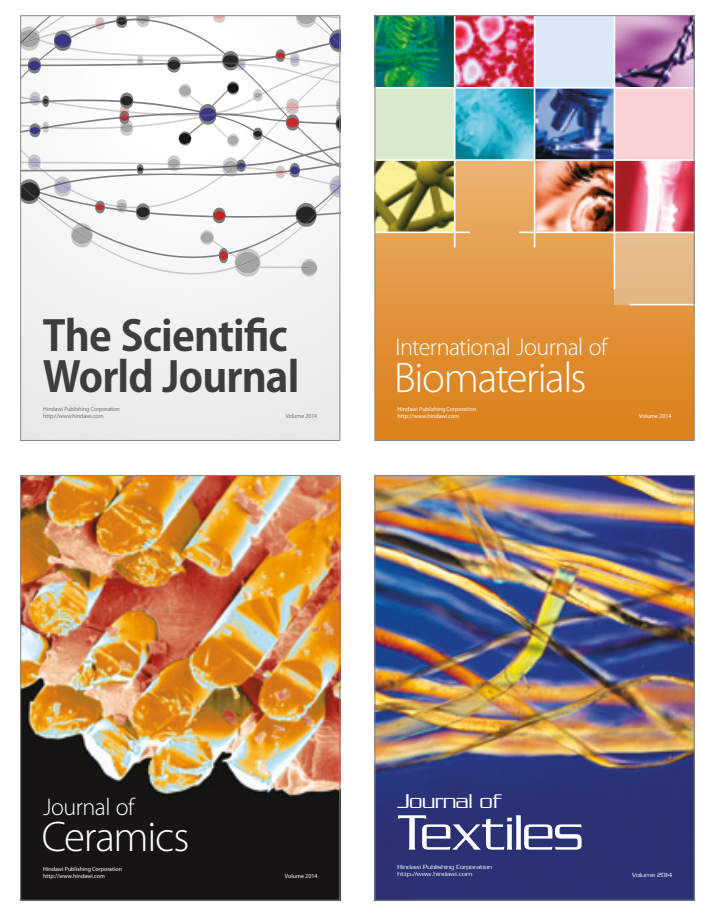